\title{
A Research on the Synchronization of Two Novel Chaotic Systems Based on a Nonlinear Active Control Algorithm
}

\author{
Israr Ahmad \\ School of Quantitative Sciences \\ University Utara Malaysia, Malaysia \\ \& College of Applied Sciences \\ Nizwa, Oman \\ iak_2000plus@yahoo.com
}

\author{
Azizan Bin Saaban \\ School of Quantitative \\ Sciences, University Utara \\ Malaysia, Malaysia \\ azizan.s@uum.edu.my
}

\author{
Adyda Binti Ibrahim \\ School of Quantitative \\ Sciences, University Utara \\ Malaysia, Malaysia \\ adyda@uum.edu.my
}

\author{
Mohammad Shahzad \\ College of Applied \\ Sciences, Nizwa, Oman \\ shehzad.niz@cas.edu.om \\ dmsinfinite@gmail.com
}

\begin{abstract}
The problem of chaos synchronization is to design a coupling between two chaotic systems (master-slave/driveresponse systems configuration) such that the chaotic time evaluation becomes ideal and the output of the slave (response) system asymptotically follows the output of the master (drive) system. This paper has addressed the chaos synchronization problem of two chaotic systems using the Nonlinear Control Techniques, based on Lyapunov stability theory. It has been shown that the proposed schemes have outstanding transient performances and that analytically as well as graphically, synchronization is asymptotically globally stable. Suitable feedback controllers are designed to stabilize the closed-loop system at the origin. All simulation results are carried out to corroborate the effectiveness of the proposed methodologies by using Mathematica 9.
\end{abstract}

Keywords-Synchronization; Lyapunov Stability Theory; Nonlinear Control; Routh-Hurwitz Criterion

\section{INTRODUCTION}

Synchronization of chaotic systems is a process where two (or many) chaotic systems eventually progress identically for different initial conditions in all future states. This means that the dynamical state of one of the system is completely dictated by the dynamical state of the other system [1]. Chaos Synchronization between two chaotic systems is one of the most primary procedures in complex systems' control and has wide potential applications in different fields [2-6]. After a pioneering work on chaos synchronization [1], synchronization of chaotic dynamical systems has received a great interest among researchers in nonlinear sciences for more than two decades [7]. Until now, diverse techniques have been proposed and applied successfully to synchronize two identical (or nearly identical) as well as nonidentical chaotic systems [8-13]. Notable among those, the Nonlinear control algorithm [7, 9] is one of the effectual techniques for synchronizing two chaotic systems [7]. Nonlinear control techniques take the advantage of the given nonlinear system dynamics to produce highperformance designs. No Lyapunov exponents or gain matrix are required for its execution. These qualities allow the designer to focus on the synchronization problem, leaving troublesome model manipulations [9].

Edward Lorenz, a meteorologist and mathematician, is known to be the pioneer of chaos theory. In the 1960s, Lorenz made his historical discovery by observing weather phenomena particularly in convections of fluids [14]. Lorenz took different mathematical models of fluid convection and simplified them into a system of ordinary differential equations and came up with a 3-D chaotic attractor for the first time, what is now known as the popular Lorenz equations [14]. After the exceptional discovery of E. Lorenz on chaotic attractor, chaos has become an interesting topic for many researchers. During the last three decades, remarkable research has been done on chaos which explored its different applications, features and fundamental properties [15].

The significance of the 3-D differential equations is that relatively simple systems could exhibit rather complex or specifically chaotic behavior. The 3-dimensional chaotic systems have many potential applications in different scientific fields such as chemical reactions, secure communications, biological systems and nonlinear circuits [15]. Due to a wide range of applications of 3-D chaotic systems, various systems such as the Chen system, Rossler system, Liu system, Qi system, Tigan system and $\mathrm{Lu}$ system [16-19] have been proposed and applied successfully to many practical systems and have shown some effective outcomes.

Recently, a new 3-D autonomous chaotic system based on a quadratic exponential nonlinear term and a quadratic cross product term has been proposed and studied [20]. A quadratic exponential nonlinear term was added to the third equation while eliminating the second term from the second equation and a nonlinear term from the third equation of the Lorenz System [20]. The new 3-D chaotic system is topologically different from the Lorenz System. The two-scroll attractor from the new system exhibits multiplex chaotic dynamics. The nonlinear dynamical properties of the new 
chaotic system such as Equilibrium points, Phase portraits, Lyapunov Exponents, Bifurcation diagram, and Poincare mapping was also extensively studied [20].

A new three dimensional chaotic system on the same construction patterns of Chen, Liu and Qi chaotic systems was proposed in [21]. An exponential term was added, the quadratic nonlinear term to the third equation of the Liu system was eliminated and a new 3-D chaotic system was proposed, topologically different from those of Chen, Liu and Qi systems and with more complex dynamics.

The main objective of this research work is to utilize the non-linear active control technique to synchronize two new chaotic systems introduced in [20, 21]. Based on the Lyapunov stability theory [22] and using the non-linear active control technique, a class of appropriate feedback controllers are designed to achieve globally asymptotic synchronization. Numerical simulations and graphs are furnished to show the performance and effectiveness of the proposed schemes.

The remainder of the paper is organized as follows: section II discusses the problem statement and the proposed methodology. In section III global chaos synchronization of two novel 3-D chaotic systems is investigated. In section IV numerical results are imparted and finally, the summary and the concluding remarks are provided in section $\mathrm{V}$.

\section{DESIGIN OF NONLINEAR ACTIVE CONTROL}

Certain synchronization algorithms belong to the driveresponse systems arrangement. The drive-response systems arrangement means that the two chaotic systems are coupled in such a manner in which the output of the response system asymptotically follows the output of the drive system and the drive system is not influenced by the exertion of the response system. Thus let us consider a drive-response systems configuration for a chaotic system given as:

$$
\dot{x}=A_{1} x+B_{1} g(x) \quad \text { (Drive system) }
$$

and

$$
\dot{y}=A_{1} y+B_{2} h(\mathrm{y})+\mu(t) \quad \text { (Response system) }
$$

where, $\quad x=\left[x_{1}, x_{1}, \ldots, x_{n}\right]^{T}, y=\left[y_{1}, y_{1}, \ldots, y_{n}\right]^{T} \in R^{n \times 1}$ are the corresponding state vectors, $A_{1}, A_{2} \in R^{n \times n}$ are the matrices and $B_{1}, B_{2} \in R^{n}$ are the vectors, $g, h: R^{n} \rightarrow R^{n}$ are the nonlinear continuous functions of the drive and response systems respectively and $\mu(t)=\left[\mu_{1}(t), \mu_{2}(t), \ldots, \mu_{n}(t)\right]^{T} \in R^{n \times 1}$ is an injected additive nonlinear controller to the controlled system.

If $g(\bullet)=h(\bullet)$ and / or $A_{1}=A_{2}, B_{1}=B_{2}$, then $\mathrm{x}$ and $\mathrm{y}$ are the states of two identical (nearly identical) chaotic systems.

If $g(\bullet) \neq h(\bullet)$ and / or $A_{1} \neq A_{2}, B_{1} \neq B_{2}$, then $\mathrm{x}$ and $\mathrm{y}$ are the states of two nonidentical chaotic systems.

The error dynamics for the synchronization of (1) and (2) can be described as:

$$
\dot{e}(t)=H(x, y, e)+\mu(t)
$$

where $H(x, y, e)$ that contains linear terms and nonlinear terms of the drive and response systems, and $e_{i}(t)=y_{i}(t)-x_{i}(t)$ is the errors dynamics. For the two (identical or nonidentical) chaotic systems in the absence of a proper controller $\quad\left(\mu_{\mathrm{i}}=0\right) \quad$ if $\quad$ the initial conditions, $\left(x_{1 d}(0), x_{2 d}(0), \ldots ., x_{n d}(0) \neq y_{1 r}(0), y_{2 r}(0), \ldots ., y_{n r}(0)\right)$, then the trajectories of the two chaotic systems will quickly bifurcate from each other in all future states and will become uncorrelated. Hence, the role of a proper feedback controller for the synchronization problem is to restrict the error dynamics converges to zero for all initial conditions [7],

i.e., $\quad \lim _{t \rightarrow \infty}\left\|e_{i}(t)\right\|=\lim _{t \rightarrow \infty}\left\|y_{i}(t)-x_{i}(t)\right\|=0$, for all $e_{i}(0) \in R^{n}$,

then the two chaotic systems (1) and (2) are said to be synchronized. Let us assume the following theorem.

Theorem 1[9]. The trajectories of the two (identical or nonidentical) chaotic systems (1) and (2) for any initial conditions $\left(\mathrm{x}_{1 \mathrm{~d}}(0), \mathrm{x}_{2 \mathrm{~d}}(0), \ldots, \mathrm{x}_{\mathrm{nd}}(0)^{1} \mathrm{y}_{1 \mathrm{r}}(0), \mathrm{y}_{2 \mathrm{r}}(0), \ldots, \mathrm{y}_{\mathrm{nr}}(0)\right)$ will be synchronized asymptotically globally with appropriate nonlinear regular stabilizing feedback controller,

$$
\mu(t)=\left[\mu_{1}(t), \mu_{2}(t), \ldots ., \mu_{n}(t)\right]^{T} \in R^{n \times 1}
$$

Proof: Let us define a candidate Lyapunov errors function as

$$
V(t)=e^{T} A e
$$

where the matrix $A=\operatorname{diag}\left(a_{1}, a_{2}, \ldots \ldots, a_{n}\right)$ is a positive definite matrix [9]. Further it is assumed that all the variables and parameters of the drive and response systems are available and measureable.

It may be noticed that $V: R^{n} \rightarrow R^{n}$ is a positive definite function by construction. It may achieve the synchronization by selecting a suitable non-linear controller ' $\mu(t)$ ' to make $\dot{V}(\mathrm{t})=-e^{T} B e$, a positive definite function (i.e., the matrix $B$ is also a positive definite matrix), then by the Lyapunov stability theory [21], the states of both drive and response systems will be asymptotically globally synchronized.

\section{SYNCHRONIZATION}

\section{A. Identical synchronization of a new chaotic system [20]}

System Description: Fey $\mathrm{Yu}$, et.al, [20] proposed and studied a new 3D autonomous chaotic system. The differential equations describing the new chaotic systems are given as: 


$$
\left.\begin{array}{rl}
\dot{x} & =a(y-x) \\
\dot{y} & =b x-c x z \\
\dot{z} & =e^{x y}-d z
\end{array}\right\}
$$

where $x, y, z \in R^{n}$ are the state variables and $a, b, c$ and $d$ are the system parameters. The new system exhibits chaotic attractor for $\mathrm{a}=10, \mathrm{~b}=40, \mathrm{c}=2$ and $\mathrm{d}=2.5$ with initial condition $(2.2,2.4,28)$

For the dynamics properties such as equilibrium points, Lyapunov exponents, phase portraits, bifurcation diagram etc for the system (5), one may refer to [20]. To achieve identical synchronization for chaotic system (5), let us consider the drive-response systems arrangement is described as:

$\left.\begin{array}{l}\dot{x}_{1}=a\left(y_{1}-x_{1}\right) \\ \dot{y}_{1}=b x_{1}-c x_{1} z_{1} \\ \dot{z}_{1}=e^{x_{1} y_{1}}-d z_{1}\end{array}\right\}$

and

$$
\left.\begin{array}{l}
\dot{x}_{2}=a\left(y_{2}-x_{2}\right)+\mu_{1} \\
\dot{y}_{2}=b x_{2}-c x_{2} z_{2}+\mu_{2} \\
\dot{z}_{2}=e^{x_{2} y_{2}}-d z_{2}+\mu_{3}
\end{array}\right\} \quad \text { (Response system) }
$$

where $x_{1}, y_{1}, z_{1} \in R^{n}$ are the state variables of the drive system with $a, b, c$ and $d$ as the system parameters and $x_{2}, y_{2}, z_{2} \in R^{n}$ as the state variables of the corresponding response system and $\mu(t)=\left[\mu_{1}(t), \mu_{2}(t), \mu_{3}(t)\right]^{T}$ as the nonlinear stabilizing feedback controller that yet to be designed.

From (6) and (7), the error dynamics can be described as:

$$
\left.\begin{array}{l}
\dot{e}_{1}=a\left(e_{2}-e_{1}\right)+\mu_{1} \\
\dot{e}_{2}=b e_{1}+c\left(x_{1} z_{1}-x_{2} z_{2}\right)+\mu_{2} \\
\dot{e}_{3}=-d e_{3}-e^{x_{2} y_{2}}+e^{x_{1} y_{1}}+\mu_{3}
\end{array}\right\}
$$

where $e_{1}=x_{2}-x_{1}, e_{2}=y_{2}-y_{1}, e_{3}=z_{2}-z_{1}$

The aim of the synchronization problem is to design a feedback controller' $\mu(t)$ ' such that:

$$
\lim _{t \rightarrow \infty}\left\|e_{i}(t)\right\|=0, \text { for all } e_{i}(0) \in R^{n} .
$$

The main focus of this section is to investigate and study the synchronization problem of (6) and (7) by designing a suitable feedback controller that when synchronizing the two chaotic systems, the effect of nonlinearity of chaotic systems does not neglect and the error signals of the two identical chaotic systems converges to the equilibrium point asymptotically globally with less control effort and sufficient transient speed. For these motivations, we assume the following theorem.

Theorem 2. The two controlled chaotic systems (6) and (7) will achieve asymptotically globally synchronization for all initial conditions $\left(x_{d}(0), y_{d}(0), z_{d}(0)\right) \neq\left(x_{r}(0), y_{r}(0), z_{r}(0)\right)$ with the following nonlinear feedback controllers:

$$
\left.\begin{array}{l}
\mu_{1}(t)=0 \\
\mu_{2}(t)=-2 b e_{1}-a e_{2}+c\left(x_{2} z_{2}-x_{1} z_{1}\right) \\
\mu_{3}(t)=e^{x_{1} y_{1}}-e^{x_{2} y_{2}}
\end{array}\right\}
$$

Proof: Replacing (9) in (8), we have:

$$
\left.\begin{array}{l}
\dot{e}_{1}=a\left(e_{2}-e_{1}\right) \\
\dot{e}_{2}=-b e_{1}-a e_{2} \\
\dot{e}_{3}=-d e_{3}
\end{array}\right\}
$$

Let us construct a Lyapunov errors function candidate as:

$$
V(e)=e_{i}^{T} A e_{i}, \text { for } i=1,2,3
$$

where:

$$
A=\left(\begin{array}{ccc}
1 & 0 & 0 \\
0 & 0.125 & 0 \\
0 & 0 & 0.5
\end{array}\right)
$$

is a positive definite matrix with $V(e)>0$ and considering $a=0, b=40, c=2$ and $d=2.5$. Now the time derivative of the Lyapunov errors function (10) is given as:

$$
\begin{aligned}
& \dot{V}(e)=2 \text { Ae } \dot{e} \Rightarrow \dot{V}(e)=2 A e_{i}\left(\dot{e}_{i}\right), \text { for } i=1,2,3 \\
& \Rightarrow \dot{V}(e)=2 A\left[e_{1}\left(\dot{e}_{1}\right)+e_{2}\left(\dot{e}_{2}\right)+e_{3}\left(\dot{e}_{3}\right)\right] \\
& \dot{V}(e)=2\left(\begin{array}{ccc}
1 & 0 & 0 \\
0 & 0.125 & 0 \\
0 & 0 & 0.5
\end{array}\right)\left[e_{1}\left(a\left(e_{2}-e_{1}\right)\right)+e_{2}\left(-b e_{1}-a e_{2}\right)+e_{3}\left(-d e_{3}\right)\right] \\
& \dot{V}(e)=-2 a e_{1}{ }^{2}-0.5 a e_{2}{ }^{2}-d e_{3}{ }^{2}=-e^{T}\left(\begin{array}{ccc}
2 a & 0 & 0 \\
0 & 0.5 a & 0 \\
0 & 0 & d
\end{array}\right) e<0
\end{aligned}
$$

i.e., $\dot{V}(e)<0$. Therefore, $\dot{V}(e)=-e^{T} B e$ and the matrix:

$$
B=\left(\begin{array}{ccc}
20 & 0 & 0 \\
0 & 10 & 0 \\
0 & 0 & 2.5
\end{array}\right)
$$


is also a positive definite matrix.

Hence, based on Lyapunov stability theory, the error dynamics (8) of the two identical chaotic systems converges to the origin asymptotically. Thus, the two chaotic systems (6) and (7) are asymptotically globally synchronized.

\section{B. Nonidentical synchronization of [20] and Lu systems}

To analyze the switching synchronization between two nonidentical chaotic systems [20] and $\mathrm{Lu}$ [19] using the Nonlinear Control Algorithm, it is assumed that the new chaotic system [20] drives the Lu Chaotic system [19]. Therefore, the drive-response systems arrangement is given as:

$$
\left.\begin{array}{l}
\dot{x}_{1}=a\left(y_{1}-x_{1}\right) \\
\dot{y}_{1}=b x_{1}-c x_{1} z_{1} \\
\dot{z}_{1}=e^{x_{1} y_{1}}-d z_{1}
\end{array}\right\}
$$

and

$$
\left.\begin{array}{l}
\dot{x}_{2}=\alpha\left(y_{2}-x_{2}\right)+\mu_{1} \\
\dot{y}_{2}=\gamma y_{2}-x_{2} z_{2}+\mu_{2} \\
\dot{z}_{2}=-\beta z_{2}+x_{2} y_{2}+\mu_{3}
\end{array}\right\} \text { (Response system) }
$$

where $x_{1}, y_{1}, z_{1} \in R^{n}$ and $x_{2}, y_{2}, z_{2} \in R^{n}$ are the corresponding state vectors of drive and response systems respectively, $a, b, c, d$ are the system parameters of the drive system and $\alpha, \beta$ and $\gamma$ are the system parameters of the response system respectively and $\mu(t)=\left[\mu_{1}(t), \mu_{2}(t), \mu_{3}(t)\right]^{T} \in R^{n \times 1}$ is the non-linear controllers. The $\mathrm{Lu}$ system describes a chaotic attractor for the parameters value, $\alpha=36, \beta=3$ and $\gamma=20$.

From (11) and (12), the error dynamics can be described as:

$$
\left.\begin{array}{l}
\dot{e}_{1}=-\alpha e_{1}+(a-\alpha) x_{1}+\alpha y_{2}-a y_{1}+\mu_{1} \\
\dot{e}_{2}=\gamma e_{2}+\gamma y_{1}-b x_{1}-x_{2} z_{2}+c x_{1} z_{1}+\mu_{2} \\
\dot{e}_{3}=-\beta e_{3}+(d-\beta) z_{1}+x_{2} y_{2}-e^{x_{1} y_{1}}+\mu_{3}
\end{array}\right\}
$$

The main objective of this part is to investigate and study the switching synchronization between (11) and (12) by designing such a feedback controller that when synchronizing the two chaotic systems, the effect of nonlinearity of chaotic systems should not be neglected and the error signal of the two nonidentical chaotic systems converges to the equilibrium point asymptotically globally with less control effort and sufficient transient speed. To achieve these goals, we presume the following theorem.

Theorem 3. The trajectories of the two chaotic systems (11) and (12) will achieve asymptotically globally synchronization for all initial conditions $\left(x_{d}(0), y_{d}(0), z_{d}(0)\right) \neq\left(x_{r}(0), y_{r}(0), z_{r}(0)\right)$ with the following control law:

$$
\left.\begin{array}{l}
\mu_{1}(t)=(\alpha-a) x_{1}-\alpha y_{2}+a y_{1} \\
\mu_{2}(t)=-2 \gamma y_{2}+\gamma y_{1}+b x_{1}+x_{2} z_{2}-c x_{1} z_{1} \\
\mu_{3}(t)=(\beta-d) z_{1}-x_{2} y_{2}+e^{x_{1} y_{1}}
\end{array}\right\}
$$

Proof: Let us construct a Lyapunov errors function candidate as: $\quad V(e)=\frac{1}{2}\left(e_{i} e_{i}^{T}\right)$, for $i=1,2,3$

Now the time derivative of the Lyapunov errors function is,

$$
\dot{V}(e)=-\alpha e_{1}{ }^{2}-\gamma e_{2}{ }^{2}-\beta e_{3}{ }^{2}=-\left(\alpha e_{1}{ }^{2}+\gamma e_{2}{ }^{2}+\beta e_{3}{ }^{2}\right)<0
$$

Therefore, $\dot{V}(e)<0$. Hence based on Lyapunov stability theory, the error dynamics of two nonidentical chaotic systems (11) and (12) converges to the origin asymptotically. Thus the two chaotic systems (11) and (12) are asymptotically globally synchronized.

\section{Identical synchronization of a new chaotic system [21]}

System Description: Recently, Chunlai Li et.al, [21] proposed and investigated a new three dimensional autonomous chaotic systems. The dynamics of the new chaotic systems is given by the following system of nonlinear differential equations,

$$
\left.\begin{array}{l}
\dot{x}=p(y-x)+y z \\
\dot{y}=(r-p) x-x z+r y \\
\dot{z}=-q z-s y^{2}
\end{array}\right\}
$$

where $x, y, z \in R^{n}$ are the state variables and $p, q, r$ and $s$ are the parameters of the new system. The new system exhibits a chaotic attractor for parameter values, $p=40, q=5, r=30$ and $s \in[0,10]$ with the Lyapunov exponents $L_{1}=3.88, L_{2}=0.00, L_{3}=-25.52$.

In this section, the aim of the study is to achieve asymptotically globally synchronization between two identical chaotic systems using a nonlinear active controller. To achieve this objective, let us consider the drive-response systems arrangement for the new chaotic system [21] which is described as:

$$
\left.\begin{array}{rl}
\dot{x}_{1} & =p\left(y_{1}-x_{1}\right)+y_{1} z_{1} \\
\dot{y}_{1} & =(r-p) x_{1}-x_{1} z_{1}+r y_{1} \\
\dot{z}_{1} & =-q z_{1}+s y_{1}^{2}
\end{array}\right\} \text { (Drive System) }
$$

and 


$$
\left.\begin{array}{l}
\dot{x}_{2}=p\left(y_{2}-x_{2}\right)+y_{2} z_{2}+\psi_{1} \\
\dot{y}_{2}=(r-p) x_{2}-x_{2} z_{2}+r y_{2}+\psi_{2} \\
\dot{z}_{2}=-q z_{2}+s y_{2}^{2}+\psi_{3}
\end{array}\right\}
$$

where $x_{1}, y_{1}, z_{1} \in R^{n}$ the state variables of the drive system with $p, q, r$ and $s$ as the system parameters and $x_{2}, y_{2}, z_{2} \in R^{n}$ the state variables of the corresponding response system and $\psi(t)=\left[\psi_{1}(t), \psi_{2}(t), \psi_{3}(t)\right]^{T} \in R^{n \times 1}$ the nonlinear active feedback controller.

From (17) and (18), the error dynamics can be described as:

$$
\left.\begin{array}{l}
\dot{e}_{1}=p\left(e_{2}-e_{1}\right)+y_{2} z_{2}-y_{1} z_{1}+\psi_{1} \\
\dot{e}_{2}=(r-p) e_{1}+r e_{2}+\left(x_{1} z_{1}-x_{2} z_{2}\right)+\psi_{2} \\
\dot{e}_{3}=-q e_{3}+s\left(y_{2}^{2}-y_{1}^{2}\right)+\psi_{3}
\end{array}\right\}
$$

$$
\text { where } e_{1}=x_{2}-x_{1}, e_{2}=y_{2}-y_{1} \text { and } e_{3}=z_{2}-z_{1} \text {. }
$$

The theme of this section is to investigate and study the synchronization of (16) and (17). To accomplish this goal, let us presume the following theorem.

Theorem 4. The trajectories of the two chaotic Systems (16) and (17) will achieve asymptotic global synchronization for any initial values $\left(x_{d}(0), y_{d}(0), z_{d}(0)\right) \neq\left(x_{r}(0), y_{r}(0), z_{r}(0)\right)$ with the following control law:

$$
\left.\begin{array}{l}
\psi_{1}(t)=y_{1} z_{1}-y_{2} z_{2} \\
\psi_{2}(t)=x_{2} z_{2}-x_{1} z_{1}-2 r e_{2} \\
\psi_{3}(t)=s\left(y_{1}^{2}-y_{2}^{2}\right)
\end{array}\right\}
$$

Proof: It is assumed that all the variables and parameters of the drive and response systems are available and measurable. Let us construct the same Lyapunov errors function as in (10) and selecting the positive definite matrix $A$ as,

$$
A=\left(\begin{array}{ccc}
\frac{1}{20} & 0 & 0 \\
0 & \frac{1}{5} & 0 \\
0 & 0 & 1
\end{array}\right)
$$

Now the time derivative of the Lyapunov errors function is,

$$
\dot{V}(e)=-4 e_{1}^{2}-12 e_{2}^{2}-10 e_{3}{ }^{2}=-e^{T}\left(\begin{array}{ccc}
4 & 0 & 0 \\
0 & 12 & 0 \\
0 & 0 & 10
\end{array}\right) e<0
$$

Therefore, $\dot{V}(e)=-e^{T} B e$ and:

$$
B=\left(\begin{array}{ccc}
4 & 0 & 0 \\
0 & 12 & 0 \\
0 & 0 & 10
\end{array}\right)
$$

is also a positive definite matrix.

Hence, based on Lyapunov stability theory, the origin of the error dynamics of the two identical chaotic systems converge to the origin asymptotically. Thus the two chaotic systems (16) and (17) are asymptotically globally synchronized.

\section{Nonidentical synchronization of [21] and Lu systems}

To achieve nonidentical synchronization for [21] using nonlinear active control strategy, it is assumed that [21] drives the $\mathrm{Lu}$ chaotic system [19]. Therefore, the drive-response systems configuration is given as;

$$
\left.\begin{array}{l}
\dot{x}_{1}=p\left(y_{1}-x_{1}\right)+y_{1} z_{1} \\
\dot{y}_{1}=(r-p) x_{1}-x_{1} z_{1}+r y_{1} \\
\dot{z}_{1}=-q z_{1}+s y_{1}^{2}
\end{array}\right\} \text { (Drive system) }
$$

and

$$
\left.\begin{array}{l}
\dot{x}_{2}=\alpha\left(y_{2}-x_{2}\right)+\psi_{1} \\
\dot{y}_{2}=\gamma y_{2}-x_{2} z_{2}+\psi_{2} \\
\dot{z}_{2}=-\beta z_{2}+x_{2} y_{2}+\psi_{3}
\end{array}\right\} \quad \text { (Response system) }
$$

where $x_{1}, y_{1}, z_{1}, x_{2}, y_{2}, z_{2} \in R^{n}$ are the corresponding state vectors of drive and response systems respectively, $p, q, r$ and $S$ are the system parameters of the drive system and $a, \beta$ and $\gamma$ are the system parameters of the response system respectively and $\psi(t)=\left[\psi_{1}(t), \psi_{2}(t), \psi_{3}(t)\right]^{T} \in R^{n \times 1}$ is the nonlinear controller that yet to be designed.

From systems of equations (19) and (20), the error dynamics can be described as;

$$
\left.\begin{array}{l}
\dot{e}_{1}=-p e_{1}-(p-a) x_{2}+\alpha y_{2}-p y_{1}-y_{1} z_{1}+\psi_{1} \\
\dot{e}_{2}=\gamma e_{2}+(\gamma-r) y_{1}-(r-p) x_{1}-x_{2} z_{2}+x_{1} z_{1}+\psi_{2} \\
\dot{e}_{3}=-q e_{3}+q z_{2}-\beta z_{2}+x_{2} y_{2}-s y_{1}^{2}+\psi_{3}
\end{array}\right\}
$$

The main contribution of this section is to achieve asymptotically globally synchronization between (19) and (20) by designing such a feedback controller that [21] is forced to track the Lu system [19] and the two systems show similar behavior for all future states. To achieve this objective let us assume the following theorem.

Theorem 5. The two chaotic systems (19) and (20) will achieve asymptotically globally synchronization for any initial values $\left(x_{d}(0), y_{d}(0), z_{d}(0)\right) \neq\left(x_{r}(0), y_{r}(0), z_{r}(0)\right) \quad$ with the following control law: 


$$
\left.\begin{array}{l}
\psi_{1}(t)=(p-a) x_{2}-\alpha y_{2}+p y_{1}+y_{1} z_{1} \\
\psi_{2}(t)=-2 \gamma y_{2}+(r+\gamma) y_{1}-(p-r) x_{1}+x_{2} z_{2}-x_{1} z_{1} \\
\psi_{3}(t)=(q-\beta) z_{2}-x_{2} y_{2}+s y_{1}^{2}
\end{array}\right\}
$$

Proof: Let us assume that the parameters of the drive and response systems are known and the states of both chaotic systems are measurable. Substituting the proposed controllers (22) in (21), we have:

$$
\begin{aligned}
& \left.\begin{array}{l}
\dot{e}_{1}=-p e_{1} \\
\dot{e}_{2}=-\gamma e_{2} \\
\dot{e}_{3}=-q e_{3}
\end{array}\right\} \\
& \Rightarrow \quad\left(\begin{array}{l}
\dot{e}_{1} \\
\dot{e}_{2} \\
\dot{e}_{3}
\end{array}\right)=\left(\begin{array}{ccc}
-p & 0 & 0 \\
0 & -\gamma & 0 \\
0 & 0 & -q
\end{array}\right)\left(\begin{array}{l}
e_{1} \\
e_{2} \\
e_{3}
\end{array}\right)
\end{aligned}
$$

It can be seen that the error system (23) is a linear system of the form, $\dot{e}=A e$. Thus by linear control theory, the system matrix $A$ is Hurwitz [23], and so the all the eigenvalues of the system matrix $A$ has negative real parts.

i.e., $\quad A=\left(\begin{array}{ccc}-40 & 0 & 0 \\ 0 & -20 & 0 \\ 0 & 0 & -5\end{array}\right)$ is Hurwitz.

Hence, the above system (23) is asymptotically stable, which implies that the two nonidentical chaotic systems (19) and (21) are synchronized asymptotically globally.

\section{NUMERICAL SIMULATIONS}

Numerical results are provided to justify the effectiveness of the proposed approaches. The parameters for [20] are taken as, $a=10, b=40, c=2$ and $d=2.5$, with initial conditions:

$$
\left(x_{1}(0), y_{1}(0), z_{1}(0)\right)=(1.2,1.2,29)
$$

and

$$
\left(x_{2}(0), y_{2}(0), z_{2}(0)\right)=(2.2,2.4,28) .
$$

The parameters for [21] are selected as, $p=40, q=5, r=30, s \in[0,10]$, with initial conditions $(3,-3,3)$ and $(-4,-7,4)$. For the Lu system, the parameters are taken as, $\alpha=36, \beta=3, \gamma=20$, with initial conditions $(6,3,4)$ and $(5,2$, 2 ). For the above chosen values, the time series of states variables for identical systems [20] and [21] is shown in Figures 1-3 \& 9-11 and for nonidentical systems [19] and ([20], [21]) in Figures 5-7 \& Figures 13-15.

Figures $4 \& 12$ illustrate the synchronization errors of two identical new chaotic systems $[20,21]$ and Figures $8 \& 16$ show the synchronization errors of two completely different chaotic systems $([20,21])$ and $\mathrm{Lu})$ respectively. For the two different chaotic systems ([20, 21] and $\mathrm{Lu}$ systems), that contain parameters mismatches and different structures, the controllers were used to synchronize the states of drive and response systems asymptotically globally when the controls are switched on at $t=0 \mathrm{~s}$. It has been shown that the new systems are forced to track the $\mathrm{Lu}$ systems and the states of two different systems show similar behavior, which shows that the error systems (Figures $8 \& 16$ ) are feedback stabilized and the investigated controllers are more robust to accidental mismatches in the transmitter and receiver. Figure 17 illustrates the derivative of Lyapunov errors functions of identical chaotic systems [20 \& 21] and nonidentical [20] and Lu Chaotic systems.

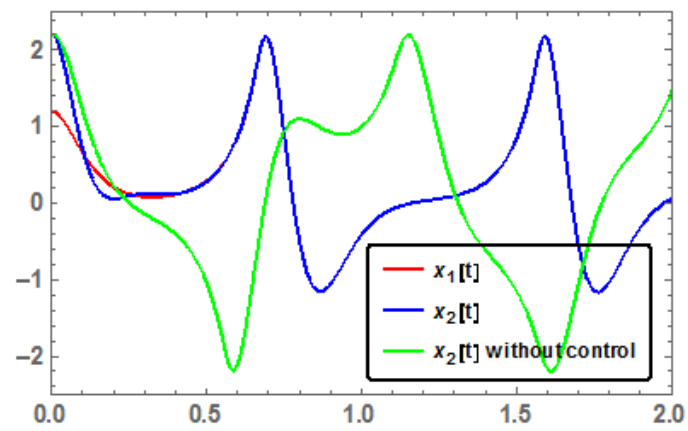

Fig. 1. Time series of states $\mathrm{x}_{1}[\mathrm{t}]$ and $\mathrm{x}_{2}[\mathrm{t}]$ (identical systems [20])

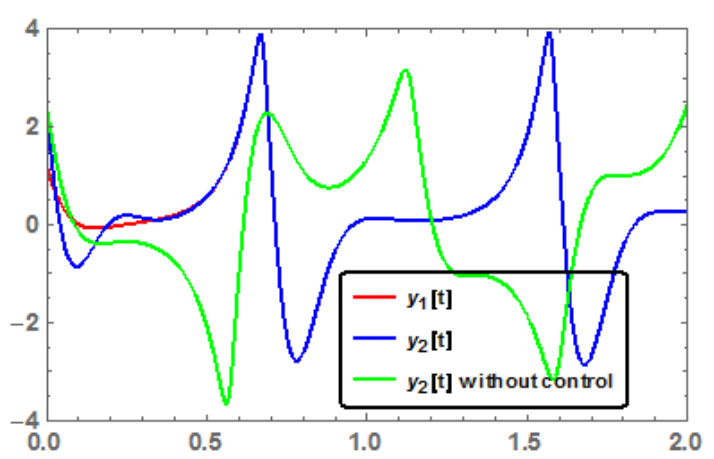

Fig. 2. Time series of states $\mathrm{y}_{1}[\mathrm{t}]$ and $\mathrm{y}_{2}[\mathrm{t}]$ (identical systems [20])

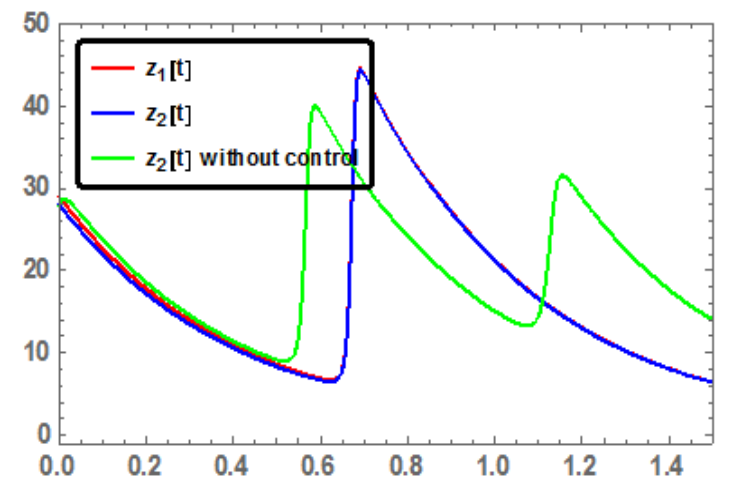

Fig. 3. Time series of states $z_{1}[t]$ and $z_{2}[t]$ (identical systems [20]) 


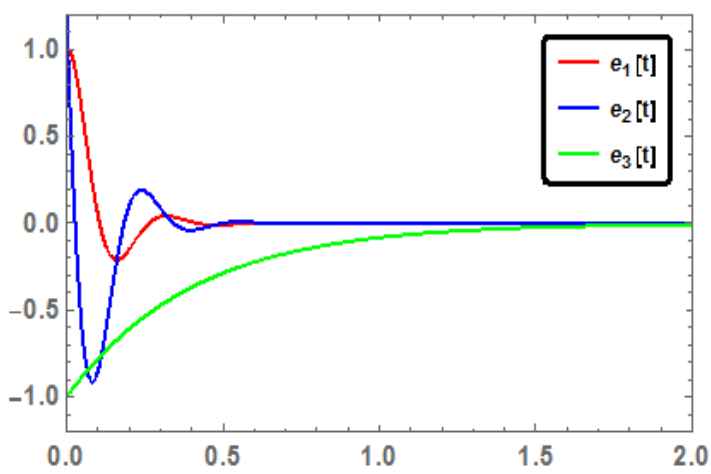

Fig. 4. Time series of error states (identical systems [20])

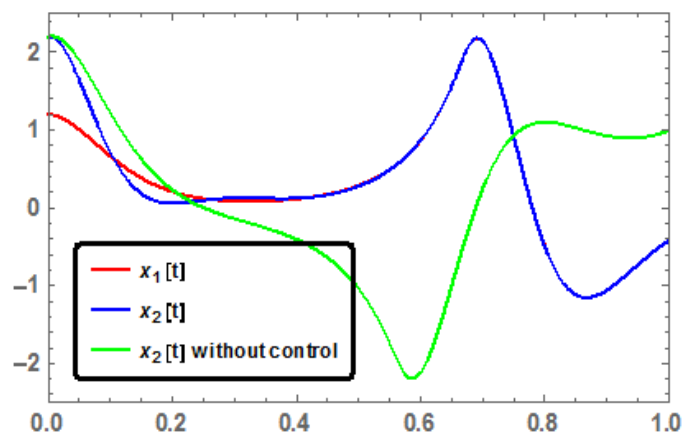

Fig. 5. Time series of $x_{1}[t]$ and $x_{2}[t]$ (non-identical systems $[19,20]$ )

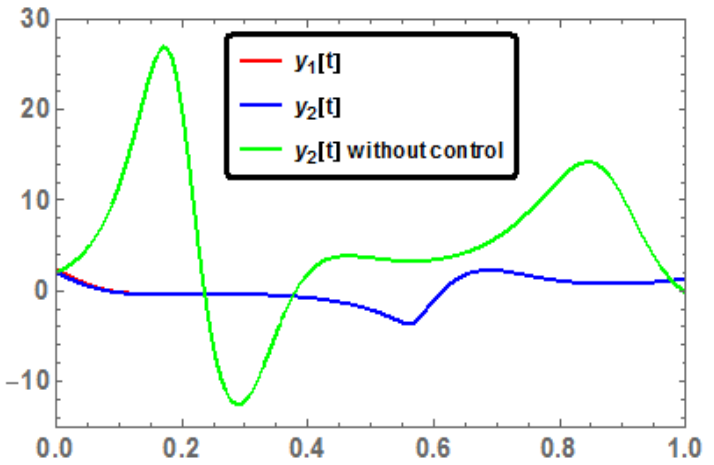

Fig. 6. Time series of $\mathrm{y}_{1}[\mathrm{t}]$ and $\mathrm{y}_{2}[\mathrm{t}]$ (non-identical systems $[19,20]$ )

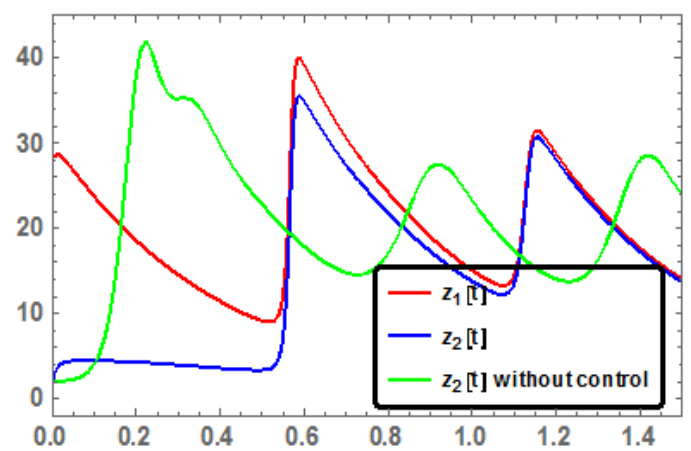

Fig. 7. Time series of $z_{1}[t]$ and $z_{2}[t]$ (non-identical systems $[19,20]$ )

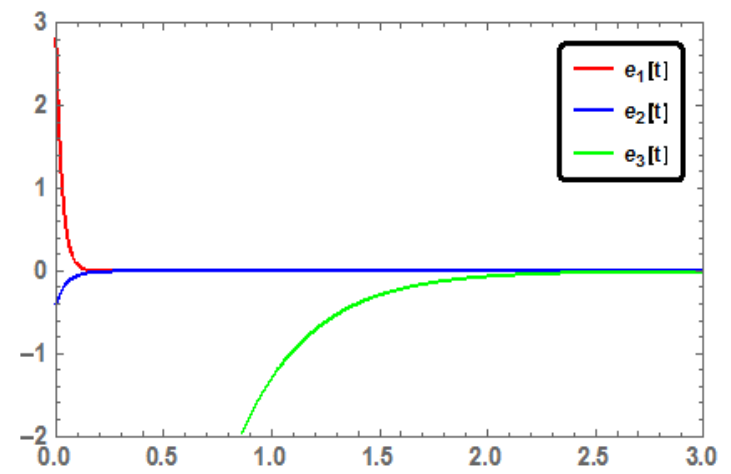

Fig. 8. Time series of error states (non-identical systems [19, 20])

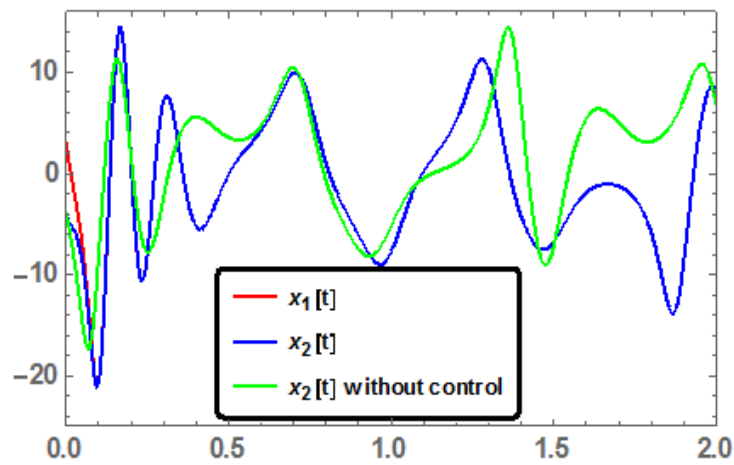

Fig. 9. Time series of $\mathrm{x}_{1}[\mathrm{t}]$ and $\mathrm{x}_{2}[\mathrm{t}]$ (identical systems [21])

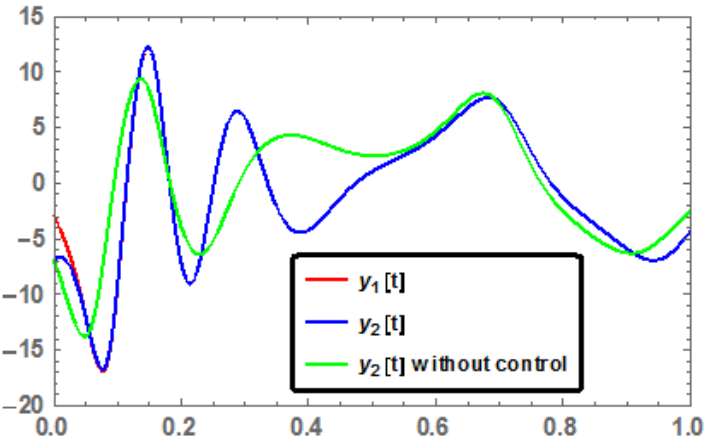

Fig. 10. Time series of $\mathrm{y}_{1}[\mathrm{t}]$ and $\mathrm{y}_{2}[\mathrm{t}]$ (identical systems [21])

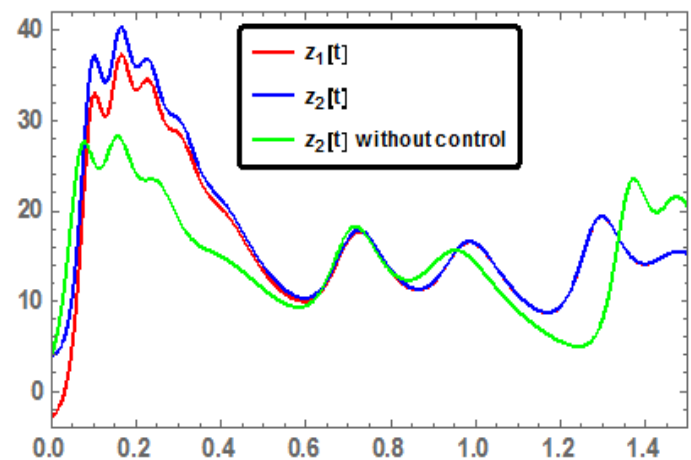

Fig. 11. Time series of $z_{1}[t]$ and $z_{2}[t]$ (identical systems [21]) 


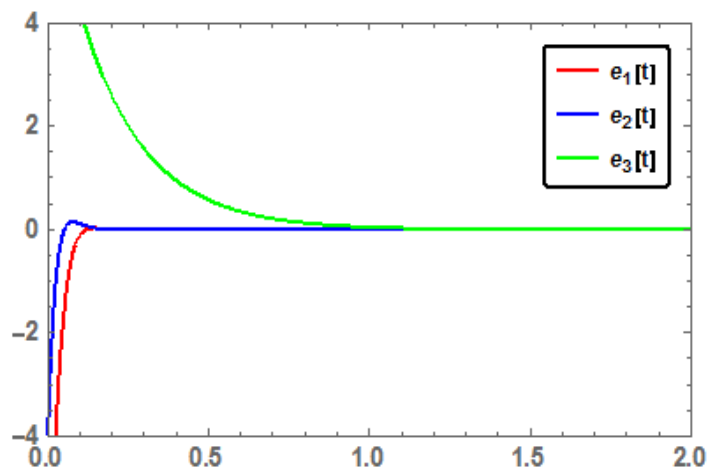

Fig. 12. Time series of error states (identical systems [21])

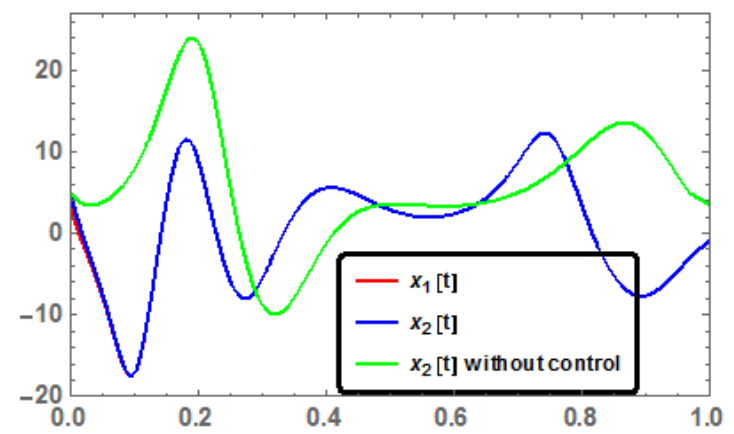

Fig. 13. Time series of $x_{1}[t]$ and $x_{2}[t]$ (non-identical systems $[19,21]$ )

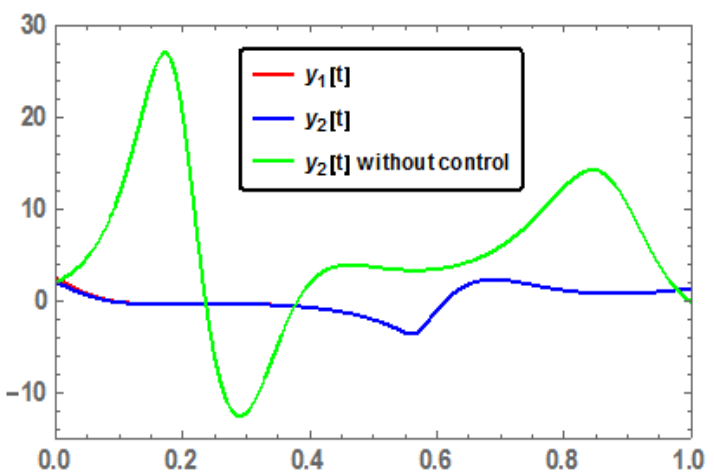

Fig. 14. Time series of $\mathrm{y}_{1}[\mathrm{t}]$ and $\mathrm{y}_{2}[\mathrm{t}]$ (non-identical systems $[19,21]$ )

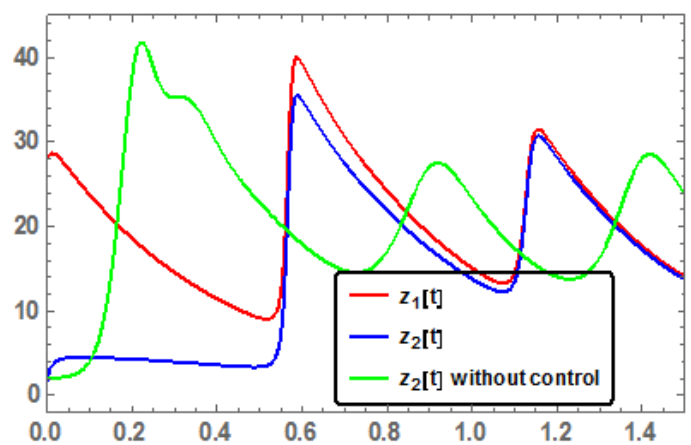

Fig. 15. Time series of $z_{1}[t]$ and $z_{2}[t]$ (non-identical systems $[19,21]$ )

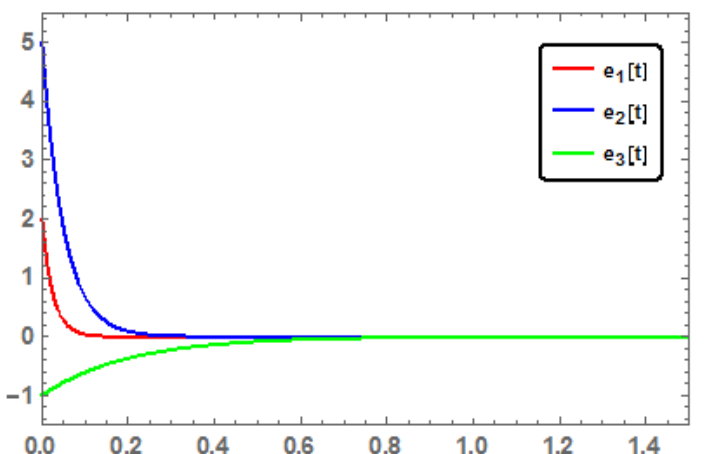

Fig. 16. Time series of error states (non-identical systems [19, 21])

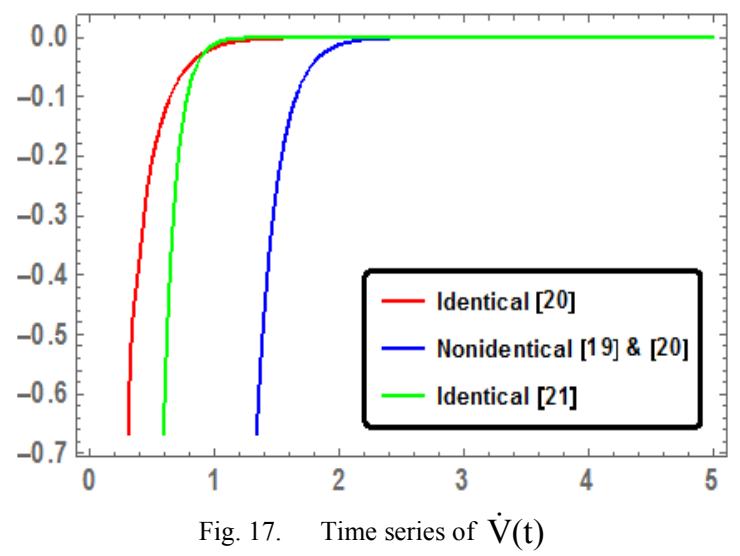

\section{CONCLUSION}

This paper investigated the global chaos synchronization of two novel chaotic systems. Based on the Lyapunov stability theory and using the Nonlinear Control Algorithm with different approaches, a class of nonlinear controllers was designed to achieve the global stability of the error dynamics. Since the Lyapunov exponents or gain matrix are not required for its execution, nonlinear control algorithm is an efficient technique to synchronize identical as well as nonidentical chaotic systems. All graphical as well as analytical results showed that the proposed schemes have excellent transient performances and that the synchronization is asymptotically globally stable. This study focused on selecting a suitable Lyapunov errors functions candidate that ensured asymptotically global stability. It was also shown that the error signals converge to the origin very smoothly with minimum rate of decay and sufficient transient speed. In addition, the synchronization with negative derivative of the Lyapunov errors functions allows large synchronizable intervals, which is significant especially for engineering applications. It should be mentioned however that in practice environmental or internal noise may provide disturbances. Hence, future work should focus on the effect of noise present on practical implementations. 


\section{ACKNOWLEDGEMENT}

The authors would like to express their gratitude to Mr. Saif Said Al Bimani (Deptt. of English, College of Applied Sciences, Nizwa, Ministry of Higher Education, Sultanate of Oman) who suggested many worthwhile changes to improve the language of the manuscript.

\section{REFERENCES}

[1] L. M. Pecora, T. L. Carroll, "Synchronization in chaotic systems". Physical Review Letters, Vol. 64, No. 8, pp. 821-824, 1990

[2] M. Shahzad, I. Ahmad, "Experimental study of synchronization \& Antisynchronization for spin orbit problem of Enceladus", International Journal of Control Science and Engineering, Vol. 3, No. 2, pp. 41-47, 2013

[3] X. F. Wang, Z. Q. Wang, "Synchronization of Chua's oscillators with the third state as the driving signal", International Journal of Bifurcation and Chaos, Vol. 8, No. 7, pp. 1599-1603, 1998

[4] K. Miyakawa, T. Okabe, M. Mizoguchi, F. Sakamoto, "Synchronization in the discrete chemical oscillation system", The Journal of Chemical Physics, Vol. 103, No. 22, pp. 9621-9625, 1995

[5] A. N. Pisarchik, F. T. Arecchi, R. Meucci, A. DiGarbo, "Synchronization of Shilnikov chaos in a $\mathrm{CO} 2$ laser with feedback", Laser Physics, Vol. 11, No. 11, pp. 1235-1239, 2001

[6] O. Moskalenko, A. A. Koronovskii, A. E. Hramov, "Generalized synchronization of chaos for secure communication: remarkable stability to noise", Physics Letters A, Vol. 374, No. 29, pp. 2925-2931, 2010

[7] A. Saaban, A. Ibrahim, M. Shahzad, I. Ahmad, "Global chaos synchronization of identical and nonidentical chaotic systems using only two nonlinear controllers", International Journal of Mathematical, Computational, Physical and Quantum Engineering, Vo. 7, No. 12, pp. 1182-1188, 2013

[8] I. Ahmad, A. Saaban, A. Ibrahim, M. Shahzad, "Global chaos identical and nonidentical synchronization of a new 3-D chaotic system using linear active control", Asia Journal of Applied Sciences, Vol. 2, No. 1 , pp. $1-12,2014$

[9] H. K. Chen, "Global chaos synchronization of new chaotic systems via nonlinear control", Chaos, Solitons \& Fractals, Vol. 23, No. 4, pp. 12451251,2005
[10] F. Yu, C. Wang, Y. Hu, J. Yin, "Projective synchronization of a fiveterm hyperbolic-type chaotic system with fully uncertain parameters", Acta Physica Sinica, Vol. 61, No. 6, pp. 0605051-0605059, 2012

[11] F. Yu, Y. Song, "Complete switched generalized function projective synchronization of a class of hyperchaotic systems with unknown parameters and disturbance inputs", Journal of Dynamic Systems, Measurement, and Control, Vol. 136, No. 1, pp. 0145051-0145056, 2014.

[12] A. Saaban, A. Ibrahim, M. Shahzad, I. Ahmad, "Identical synchronization of a new chaotic system via nonlinear control and linear active control techniques: a comparative analysis", International Journal of Hybrid Information Technology Vol.7, No.1, pp. 211-224, 2014

[13] I. Ahmad, A. Saaban, A. Ibrahim, M. Shahzad, "Global chaos synchronization of two different chaotic systems using nonlinear control", International Journal of Sciences: Basic and Applied Research, Vol. 14, No. 1, pp. 225-238, 2014

[14] E. Lorenz, "Deterministic nonperiodic flow", Journal of the Atmospheric Sciences, Vol. 20, No. 2, pp. 130-141, 1963

[15] S. Boccaletti, C. Grebogi, Y. C. Lai, H. Mancini, D. Maza, "The control of chaos: theory and applications", Physics Reports, Vol. 329, No. 3, pp. 103-109, 2000

[16] L. S. Tee, Z. Salleh, "Dynamical analysis of a modified Lorenz system", Journal of Mathematics, Vol. 2013, Article ID 820946, 2013

[17] G. Qi, G. Chen, A. A. van Wyk, B. J. van Wyk, Y. Zhang, "A four-wing chaotic attractor generated from a new 3-D quadratic autonomous system", Chaos, Solitons \& Fractals, Vol. 38, No. 3, pp. 705-721, 2008

[18] G. Tigan, D. Opris, "Analysis of a 3D chaotic system", Chaos, Solitons \& Fractals, Vol. 36, No. 5, pp. 1315-1319, 2008

[19] J. Lu. G. Chen, "A new chaotic attractor coined", International Journal of Bifurcation and Chaos, Vol. 12, No. 3, pp. 659-662, 2002

[20] F. Yu, C. Wang, "A novel three dimensional autonomous chaotic system with a quadratic exponential nonlinear term", Engineering, Technology \& Applied Science Research, Vol. 2, No. 2, pp. 209-215, 2012

[21] C. Li, L. Wu, H. Li, Y. Tong, "A novel chaotic system and its topological horseshoe", Nonlinear Analysis: Modelling and Control, Vol. 18, No. 1, pp. 66-77, 2013

[22] H. K. Khalil, Non Linear dynamical Systems. Prentice Hall, $3^{\text {rd }}$ edi, NJ, 07458, USA, 2002

[23] R. C. Dorf, R. H. Bishop, Modern Control Systems, 9th Ed. Princeton Hall, USA, 2001 